\title{
The Canadian 24-Hour Movement Guidelines for Children and Youth: Implications for practitioners, professionals, and organizations ${ }^{1}$
}

\author{
Amy E. Latimer-Cheung, Jennifer L. Copeland, Jonathon Fowles, Lori Zehr, Mary Duggan, \\ and Mark S. Tremblay
}

\begin{abstract}
The new Canadian 24-Hour Movement Guidelines for Children and Youth emphasize the integration of all movement behaviours that occur over a whole day (i.e., light, moderate, and vigorous physical activity, sedentary behaviour, and sleep). These guidelines shift the paradigm away from considering each behaviour in isolation. This concept of the "whole day matters" not only calls for a change in thinking about movement but also for redevelopment of dissemination and implementation practice. Past guideline launch activities largely have aimed to create awareness through passive dissemination strategies (e.g., Website posts, distribution of print resources). For the integrated guidelines to have public health impact, we must move beyond dissemination and raising of awareness to implementation and behaviour change. Shifting this focus requires new, innovative approaches to intervention, including interdisciplinary collaboration, policy change, and refocused service provision. The purpose of this paper is to identify practitioners, professionals, and organizations with potential to disseminate and/or implement the guidelines, discuss possible implementation strategies for each of these groups, and describe the few resources being developed and those needed to support dissemination and implementation efforts. This discussion makes readily apparent the need for a well-funded, comprehensive, long-term dissemination, implementation, and evaluation plan to ensure uptake and activation of the guidelines.
\end{abstract}

Key words: implementation, dissemination, awareness, integrated guidelines, physical activity, sedentary behaviour, sleep.

Résumé : Les nouvelles Directives canadiennes en matière de mouvement sur 24-heures à l'intention des enfants et des jeunes mettent l'accent sur tous les comportements kinésiques d'une journée entière (c.à-d. activite physique dintensite legere, moderee et vigoureuse; sedentarite, et sommeil). Ces directives mettent de côté le paradigme qui considère les comportements de façon isolée. Le concept " une affaire de toute une journée » ne nous invite pas seulement à modifier notre perception du mouvement, mais aussi à réfléchir à nos modes de diffusion du message et de la mise en œuvre des pratiques. Les activités de lancement des directives antérieures étaient axées sur la sensibilisation au moyen de stratégies passives de diffusion (p. ex. publications sur le web, distribution de documents imprimés). Nous devons passer du stade de la diffusion et de la sensibilisation à la mise en application et à la modification de comportement afin que ces directives intégrées aient un impact sur la santé publique. Cette nouvelle perspective exige une approche novatrice sur le plan de l'intervention incluant des collaborations interdisciplinaires, des modifications des politiques et des modifications de l'offre de service. Cet article a pour objectif d'identifier des praticiens, des professionnels et des organismes disposant du potentiel de diffusion et/ou d'application des directives, de traiter des stratégies de mise en œuvre de la part de ces groupes, de décrire les quelques ressources en développement et les ressources requises pour la diffusion et la mise en œuvre. Cet article souligne le besoin de plans solides, approfondis et à long terme de diffusion, de mise en œuvre et d'évaluation pour s'assurer de l'adoption et de l'activation des directives. [Traduit par la Rédaction]

Mots-clés : mise en œuvre, diffusion, sensibilisation, directives intégrées, activité physique, comportement sédentaire, sommeil.

\section{Introduction}

Evidence suggests that current levels of physical activity, sedentary behaviour, and sleep among Canadian children and youth are cause for concern (Colley et al. 2011, 2012; ParticipACTION 2016;
Tremblay et al. 2014). Further evidence provided in this journal supplement demonstrates important health-related benefits of increased childhood physical activity (Poitras et al. 2016), decreased sedentary behaviours (especially screen time) (Carson et al. 2016a), and the maintenance of adequate sleep (Chaput et al.

Received 17 February 2016. Accepted 4 April 2016.

A.E. Latimer-Cheung. School of Kinesiology and Health Studies, Queen's University, 28 Division Street, Kingston, ON K7L 3N6, Canada; Healthy Active Living and Obesity Research Group, Children’s Hospital of Eastern Ontario Research Institute, 401 Smyth Road, Ottawa, ON K1H 8L1, Canada.

J.L. Copeland. Deptartment of Kinesiology and Physical Education, University of Lethbridge, 4401 University Drive, Lethbridge AB T1K 3M4, Canada.

J. Fowles. School of Kinesiology, Acadia University, 550 Main Street, Wolfville, NS B4P 2R6, Canada.

L. Zehr. Camosun College, 3100 Foul Bay Road, Victoria, BC V8P 5J2, Canada.

M. Duggan. Canadian Society for Exercise Physiology, 370-18 Louisa St., Ottawa, ON K1R 6Y6, Canada.

M.S. Tremblay. Healthy Active Living and Obesity Research Group, Children's Hospital of Eastern Ontario Research Institute, 401 Smyth Road, Ottawa,

ON K1H 8L1, Canada; Department of Pediatrics, University of Ottawa, 401 Smyth Road, Ottawa, ON K1H 8L1, Canada.

Corresponding author: Amy Latimer-Cheung (email: amy.latimer@queensu.ca).

1This paper is part of a Special issue entitled Canadian 24-Hour Movement Guidelines for Children and Youth: An Integration of Physical Activity, Sedentary Behaviour, and Sleep.

Copyright remains with the author(s) or their institution(s). This work is licensed under a Creative Commons Attribution 4.0 International License (CC BY 4.0), which permits unrestricted use, distribution, and reproduction in any medium, provided the original author(s) and source are credited. 
2016). New and innovative analyses have recently demonstrated that the combination of movement/non-movement behaviours (sleep, sedentary behaviours, and physical activity) matters for health-related indicators in children and youth (Carson et al. 2016b; Chaput et al. 2014; Saunders et al. 2016). This collective information was the impetus for the development of the Canadian 24-Hour Movement Guidelines for Children and Youth: An Integration of Physical Activity, Sedentary Behaviour and Sleep - the world's first "integrated" movement guidelines (Tremblay et al. 2016a). The guidelines, described fully by Tremblay and colleagues (2016a) in this journal supplement, provide evidence-based recommendations for a healthy day, including a combination of ight, moderate, and vigorous physical activity, sedentary behaviours, and sleep. The new guidelines represent a paradigm shift for practitioners, professionals, and organizations. With an emphasis on the integration of all behaviours and moving away from considering each behaviour in isolation (Tremblay et al. 2016b), the new guidelines call for a change in thinking about movement and adaptations to dissemination and implementation practice. Although changes in thought and practice can be daunting, there are notable benefits to adopting the new guidelines.

It has been 5 years since the present Canadian Physical Activity Guidelines for Children and Youth and the Canadian Sedentary Behaviour Guidelines for Children and Youth were released. The dissemination of the new integrated guidelines will draw public attention back to recommended amounts of movement for children and youth and will provide practitioners, professionals, and organizations with updated, refreshed, and novel messages for dissemination. Increased message novelty is an effective strategy to increase the impact of public health messages (Louis and Sutton 1991). Moreover, the integrated guidelines create opportunity for a consolidated single message about movement - rather than separate messages for physical activity, sedentary behaviour, and sleep. A consolidated message creates efficiencies in disseminating information about multiple behaviours through a single resource and may also facilitate message recall by simplifying multiple recommendations into 1 message. The integrated guidelines also create opportunity to leverage public interest in 1 movement behaviour to direct attention to another. For example, awareness of physical activity guidelines far exceeds awareness of sedentary behaviour guidelines for children and youth (LeBlanc et al. 2015). With an integrated guideline, individuals seeking information about physical activity recommendations for children and youth will also be introduced to information about sedentary behaviour and sleep.

In recognizing the whole day as important (an obvious but often ignored reality in practice), the integrated guidelines open new avenues for intervention. For example, a practitioner may begin counselling an inactive child to become more active by encouraging the child to get a good night's sleep, which in turn may result in the child waking-up feeling refreshed and receptive to physical activity. The range of movement behaviours included in the guidelines allows organizations to develop programs and services that are tailored to their capacity. Previously, in constrained context where opportunity for moderate to vigorous physical activity was limited (e.g., a classroom), physical activity guidelines may have been disregarded because the opportunity for movement was minimal. More inclusive recommendations broaden the scope of possible interventions and makes movement possibilities more inclusive, probable, and feasible. For example, a classroom teacher could plan for frequent breaks of light physical activity through the day to limit sitting time.

Of further benefit, the integrated guidelines create opportunity for a coordinated collaborative approach to child and youth health. Whereas sleep and physical activity organizations normally exist in silos, collaboration between these organizations may be particularly beneficial and provides an opportunity for previously untapped partnerships and message cross-promotion. This could include the sharing of tools and resources, the collaborative development of interdisciplinary educational materials, and possible partnerships for funding opportunities. Organizations may also benefit from developing new or expanded referral networks to optimize client services. In light of the breadth of the guidelines, collaborations can expand beyond health- and movement-oriented organizations (e.g., YM/YWCA, physical education associations, municipal sports and recreation) to general service organizations for children and youth (e.g., Scouts Canada or Girl Guides of Canada) that may be able to address several areas of the guidelines through their programming. Engaging more organizations is strategic. It will broaden the reach of the guidelines, facilitate earlier uptake, and increase the likelihood of longterm adoption.

Given the clear benefits of adopting the guidelines, a coordinated, adequately resourced and sustained dissemination and implementation strategy is necessary. Currently, only a plan for initial guideline launch and limited resource development is formally in place. Planned launch activities and resources being development are listed in Table 1 . Novel to this launch, however, the guidelines have a visual identity that was developed in collaboration with a creative agency. The identity is meant to provide a uniform look to all guideline materials that enhances recognition, recall, and understanding of the guidelines. The development of this visual identity is indicative of the enthusiastic support of the integrated guidelines by funders and suggests promise of a more comprehensive dissemination and implementation strategy; however, as of June 2016, financial resources for long-term dissemination and implementation strategy remain elusive.

Guideline promotion activities have traditionally been underresourced, severely limiting the scope of dissemination activities and precluding entirely guideline implementation efforts, which consequently blunted the impact of new guidelines. Specifically, in the absence of adequate resources we have relied on passive dissemination strategies to deliver new guidelines to practitioners, professionals, and organizations that in turn communicate the guidelines within their networks. The primary goal of these dissemination activities is to create guideline awareness. Of concern, awareness is an outcome with limited public health impact. Within health communications theory (e.g., hierarchy of effects model; McGuire 1984), awareness is a distal precursor to behaviour change. Once aware of guidelines, several cognitive changes are necessary to eventually lead to behaviour change - the ultimate goal. Given that unprompted awareness of the physical activity and sedentary behaviour guidelines for children and youth is low (ranging from 3\%-12\%; LeBlanc et al. 2015), it is not surprising that the majority of children and youth do not meet the guidelines. The emphasis on awareness also has limited the range of dissemination strategies used heavily focusing on educational interventions (e.g., creation of informational resources, Webinars) and communications and marketing. Failure to adequately fund a comprehensive dissemination and implementation strategy for the integrated guidelines creates potential for similar, disappointing outcomes as observed in previous guideline initiatives. Adequate resources would allow for the reorientation of efforts to not only address awareness through guideline dissemination but to emphasize implementation through meaningful participation of all stakeholders, resulting in activation of the guidelines. Shifting this focus would result in new approaches to interventions, including interdisciplinary collaboration, policy change, environmental restructuring, and refocused service provision (Michie et al. 2011).

This paper is a step towards envisioning a comprehensive plan for disseminating and implementing the integrated guidelines. It also is meant to be a starting point to stimulate the healthy active living sector to explore ways to implement and activate the new guidelines. We discuss how practitioners, professionals, and orga- 
Table 1. Dissemination and implementation resources and activities developed/planned or needed.

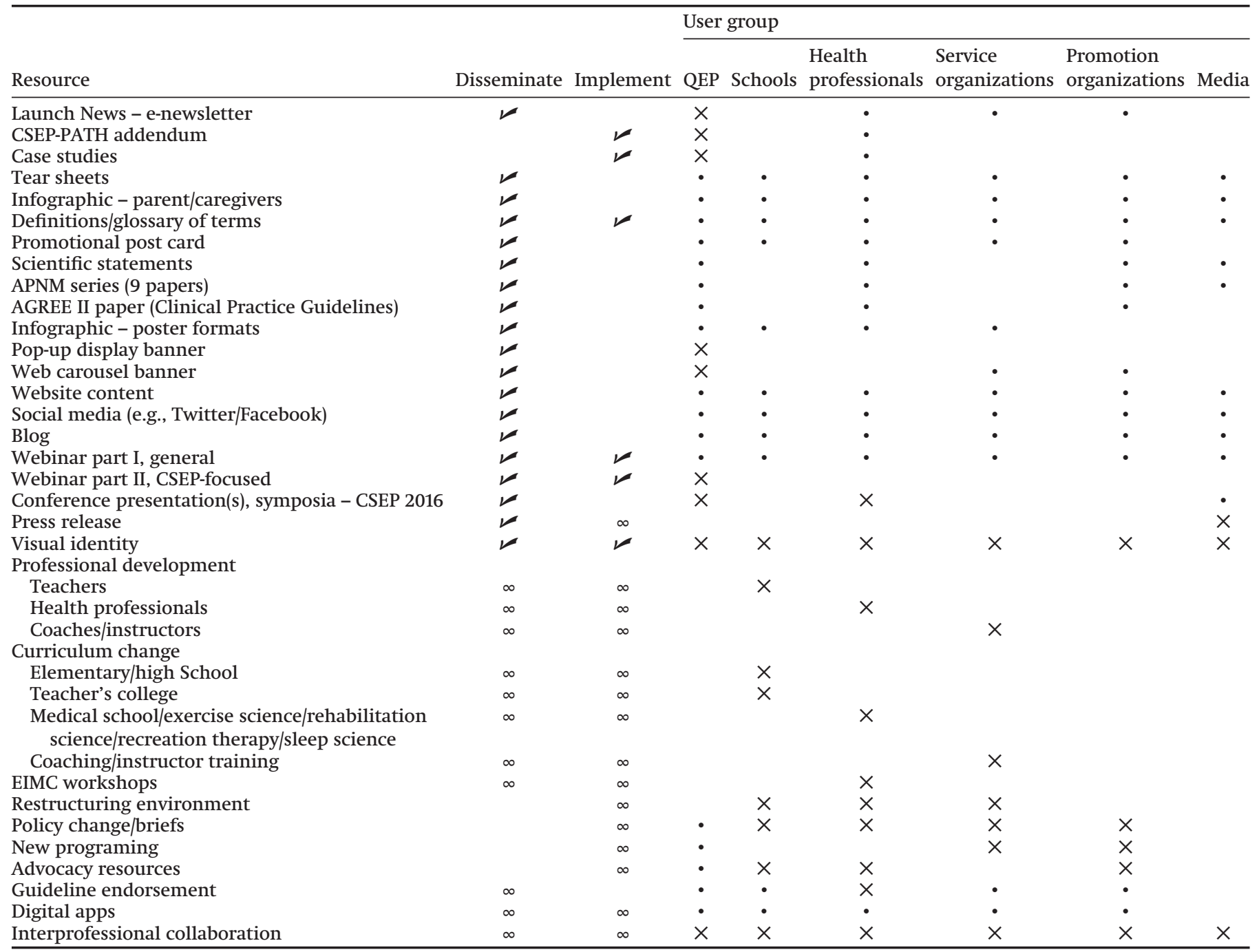

Note: $\boldsymbol{\bullet}$, resource/activity developed or planned; $\infty$, resource/activity needed; $\times$, target user group; $\bullet$, secondary user group (i.e., resource/activity not tailored to the user group but still relevant). AGREE, Appraisal of Guidelines for Research Evaluation; APNM, Applied Physiology, Nutrition, and Metabolism; CSEP, Canadian Society for Exercise Physiology; CSEP-CEP, CSEP Certified Exercise Physiologists; CSEP-CPT, CSEP Certified Personal Trainers; CSEP-PATH, CSEP-Physical Activity Training for Health; EIMC, Exercise is Medicine Canada; QEP, Qualified Exercise Professional including CSEP-CEP, CSEP-CPT, CSEP Instructor/Examiner.

nizations can be involved in the broad dissemination and implementation of the new integrated movement guidelines. Many of the suggestions are preliminary but are nonetheless important. Simply moving beyond building awareness will represent innovation in the dissemination and implementation of Canadian movementrelated guidelines. Further innovation will come as stakeholders, researchers, and relevant experts (e.g., staff from marketing agencies, Web designers, app developers) adapt and activate the preliminary ideas we put forth.

\section{Framework for change}

When considering how to optimize guideline dissemination and implementation, the Health Promotion Research Centre (HPRC) framework (Harris et al. 2012; see Fig. 1) for disseminating evidence-based health promotion practices provides a useful guide. The framework identifies 3 main actors in the dissemination and implementation process: (i) researchers: the team that creates new knowledge to inform best practices, (ii) disseminators: organizations that lead the dissemination process, and (iii) user organizations: the groups that implement the practices putting the guidelines into place. For successful dissemination, researchers and disseminators should work collaboratively to develop ap- proaches to disseminate the health promotion practice to user groups. In developing these approaches, researchers and disseminators must consider the needs, capacity, and readiness of the user organization through an assessment of "linkages and learnings". In turn, implementation of the practice within an organization entails a series of steps beginning with adoption, implementation, maintenance, and culminating in change in organization and professional practice (Harris et al. 2012).

The integrated guidelines provide an interesting context for application of the HPRC framework. In terms of actors, the researchers include members of the guideline development team. The designated lead disseminators are the Canadian Society for Exercise Physiology (CSEP) and ParticipACTION (Canada's national voice for physical activity). They have been designated the lead disseminators by the leadership team, including representation from the Healthy Active Living and Obesity Research Group, CSEP, ParticipACTION, the Public Health Agency of Canada, and the Conference Board of Canada. While all of these organizations are extensively supporting dissemination and implementation of the new guidelines, CSEP and ParticipACTION are responsible for liaising with user groups and for creating resources for dissemination and implementation. It is these activities that are described 
Fig. 1. Health Promotion Research Centre Framework from Harris et al. 2012. () The Government of the United States of America. The work is in the public domain and can be reproduced without permission.

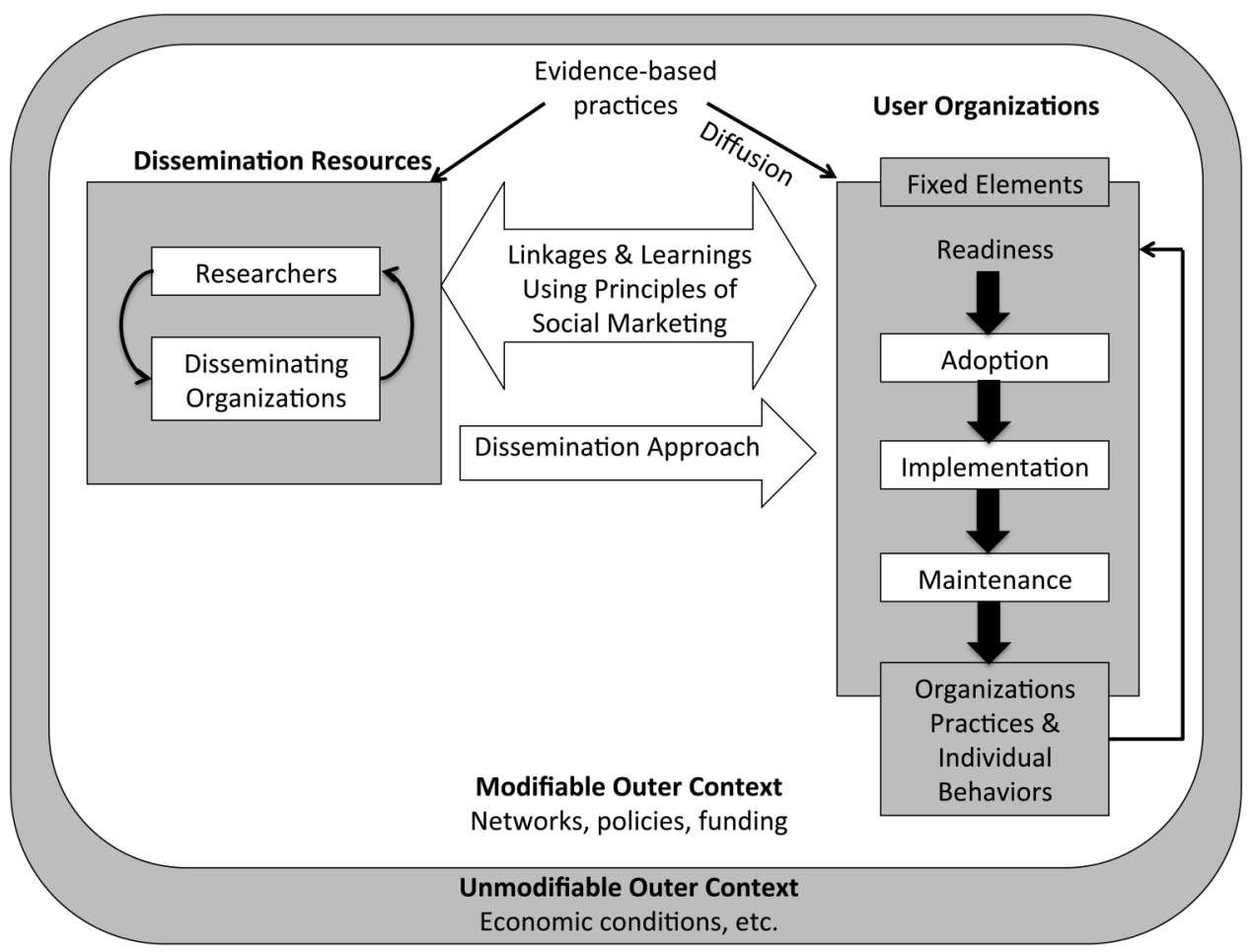

in subsequent sections of this paper and thus only CSEP and PartcipACTION are featured.

While CSEP and ParticipACTION can undertake activities to directly disseminate the guidelines to the target audience - children and youth and their parents and caregivers - a more efficient strategy is to engage intermediary organizations and groups of practitioners and professionals that serve the target audience (Brawley and Latimer 2007). As such, these groups take on a role as disseminators. Beyond dissemination and building awareness, many intermediaries also are well positioned to serve as user groups. In this role, user groups implement and activate the guidelines through their services and programs.

Any group involved in research dissemination, public education and awareness, program delivery or advocacy in the broad healthy active living sector and the child and youth development sector can play an important role in the dissemination and implementation of these new guidelines. Developing an exhaustive list of practitioners, professionals, and organizations to target is not feasible, and almost sure to inadvertently exclude some important groups, especially considering the multidisciplinary nature of the new guidelines. Below we identify a sample of key groups with potential to disseminate and/or implement the guidelines. We discuss the implications of the guidelines for these groups as a preliminary assessment of linkages and learnings, describe resources (if any) being developed to support each group's dissemination and implementation efforts, and provide suggestions that should be further explored in the development of a comprehensive dissemination and implementation plan, if and when resources become available.

\section{Opportunities for guideline dissemination and implementation}

\section{Exercise professionals}

\section{Dissemination}

Qualified exercise professionals, such as CSEP Certified Personal Trainers ${ }^{\circledR}$ (CSEP-CPTs) and Certified Exercise Physiologists ${ }^{\circledR}$
(CSEP-CEPs), play an important role educating the public. They often provide general advice about healthy lifestyles that expands beyond just physical activity to include things such as minimizing sedentary behaviour and the importance of adequate sleep for recovery. As such, many of these professionals will find that the new guidelines support the information they are already providing to clients. For others, the guidelines may require them to expand their practice to incorporate an understanding of the importance of movement behaviours across the entire day and how these different behaviours interact to support a more holistic view of health and wellness. It is important to note that not all exercise professionals work directly with children and youth, and in fact, working with clients under the age of 15 years is outside of the scope of practice for many CSEP-CPTs. However, many CSEPCEPs work with young clients and some CSEP-CPTs have additional education and training to work with children and youth. Furthermore, both are likely to have clients who are parents that may have questions about the new guidelines or may be working in settings such as community centres or healthcare teams where other intermediaries consult them for guidance. Thus, it is important that all exercise professionals understand the integrated guidelines, and that they play a key role in helping with the dissemination of the message that the combination of movement behaviours over the whole day is important.

To facilitate these dissemination activities, CSEP is creating a 3-part series of an electronic news update: "24-hr Guidelines Launch News". Launch News Issue \#1 was released prior to the launch of the new guidelines and was targeted to current CSEP instructor/examiners, and CSEP certified members as well as other exercise and health promotion professionals. This was a teaser to promote the launch and associated tools. Issue \#2 was distributed with the guidelines release to a wide audience to promote the special issue of Applied Physiology, Nutrition, and Metabolism, and contained all of the background research papers, media release, and prepared informational resources. Issue \#3 is planned for Fall 2016 to profile a new Workbook for CSEP professionals, promote available learning opportunities, and profile how the guidelines 
are being adopted and used by practitioners, professionals, and organizations. In addition to these resources, CSEP will also be working towards updating current tools and resources to reflect the information and content of the new guidelines, and will encourage other organizations to do the same. The updated resources will include 1-page tear sheets, an infographic, and Website content.

\section{Implementation}

Qualified exercise professionals who are working with children and youth are optimally positioned to activate the guidelines beyond the simple sharing of information. Implementing the new guidelines in their practice will require qualified exercise professionals to take a more holistic approach to both assessment and prescription. While assessment typically focuses on physical activity participation and/or physical fitness, the new integrated guidelines mean that an assessment of sleep, sedentary time, light physical activity, and/or total physical activity is also warranted. Qualified exercise professionals can then use information about daily movement and sleep patterns to inform individualized lifestyle advice. For example, in addition to providing an exercise prescription, they should also incorporate goals for sedentary and sleep time into programs they develop for younger clients. They can recommend strategies to incrementally change behaviours, and they can help children and youth achieve the targets in the guidelines using tools such as daily logs for tracking sleep and screen time, and pedometers for tracking daily steps. Qualified exercise professionals who are working with younger clients will also want to engage parents and caregivers to have them support their recommendations as much as possible, both by encouraging adherence and by modeling similar behaviours.

In terms of resources to support qualified exercise professionals' implementation of the guidelines, various tools have already been developed or are in the process of development (see Table 1). The Physical Activity and Sedentary Behaviour Questionnaire can be used to assess sedentary time and physical activity in youth ages 12-17 years (CSEP 2013). Assessment tools for children under 12 years of age and additional tools for assessing sleep or the combination of behaviours over $24 \mathrm{~h}$ will need to be developed, and qualified exercise professionals may also need to enlist parents and caregivers to help them assess movement behaviours in younger children.

CSEP is developing a CSEP-Physical Activity Training for Health (CSEP-PATH®) "Workbook" addendum for exercise and health professionals and CSEP certification candidates that will serve as the foundation document for professionals' ancillary materials (e.g., client assessment tools, educational Webinars, and client handouts). This workbook will include an "Introduction/How to Use" section, the 24-h movement guideline scientific statements, and messaging materials for clients (e.g., tear sheets and infographic, a list of definitions, several case studies, along with links to public-facing content available online). The list of tools and resources that need to be updated is extensive, and it will be an ongoing process. An update workshop for all CSEP Instructor Examiners will be available in person at the 2016 CSEP Annual General Meeting (Victoria, B.C., Canada; October 2016) and as a Webinar following the meeting, with accompanying instructor slides to be used in workshops and classes. Content from the workbook will need to be included in the course content supporting the respective certifications, and the new guidelines and materials will be integrated into workshops with healthcare professionals that are on-going through the CSEP Exercise is Medicine ${ }^{\circledR}$ Canada (EIMC) initiative.

\section{The school system}

\section{Dissemination}

Schools are a prime intermediary for dissemination as they have extensive reach of the target population - children and youth - and have established mechanisms for reaching parents, caregivers, teachers, administrators, and trustees. The guidelines could be disseminated passively to students and their parents through print resources with information about the guidelines. More desirable are active dissemination strategies that would involve incorporating the guidelines into physical education, health education, or home economics curricula. A passive dissemination approach would have little impact on the school system, assuming print resources are created by the lead disseminators, CSEP, and ParticipACTION, and paid through funds allocated by the integrated guideline leadership team. Active dissemination requires curriculum change and professional development for teachers to gain understanding of the guidelines.

CSEP and ParticipACTION recognize these needs and have developed some tools to facilitate guideline dissemination through the school system. Tear sheets and infographics are available for dissemination to parents and caregivers. However, there is no formal plan for coordinated dissemination of these resources through the school system. To provide a foundation for active dissemination, teachers and administrators will be invited to attend CSEP-hosted Webinars about the guidelines as a form of professional development. More formal forms of professional development could occur during designated professional development days or at education conferences (e.g., Physical and Health Education (PHE) Canada's Annual conference). The new guidelines also should be integrated into new teacher training activities. Provincial and territorial ministries of education must mandate changes to the elementary and high school curriculum to facilitate active dissemination of the guidelines to students. This may necessitate advocacy efforts from stakeholder groups (e.g., parent groups, teacher associations, physical education organizations) to create pressure for change.

\section{Implementation}

Because physical activity and sedentary behaviour are important considerations in the structuring of the school day, schools are well suited to implement the guidelines. Schools have had limited success implementing the guidelines in the past (Stone et al. 2012) but now with a broader range of behaviours to choose from, the guidelines may be better suited to be incorporated into the school setting. To fully activate the guidelines, policy change is necessary to increase opportunity for increased light, moderate, and vigorous physical activity and reduced sitting time. Stretch breaks, nonsitting time, and physical activity movement bursts should occur throughout the day, not just in scheduled physical education or recess blocks. School start times also should be evaluated to ensure adequate sleep patterns. A strategy to address identified barriers to physical activity participation arising from liability and perceived safety concerns along with limited access to facilities should be developed. The guidelines also could be activated through creative curriculum changes that not only build awareness of the guidelines but also teach students how to implement the guidelines. Such changes need not be limited to traditional subject areas (e.g., physical education) but should pervade all aspects of the curriculum (e.g., the guidelines could be integrated in to a math lesson about proportions) and the whole school environment. Enhancing active transportation initiatives are also important opportunities that may be leveraged with school boards and community partners (Larouche et al. 2014). The 24-h guidelines are also conceptually congruent with the comprehensive school health approach and opportunities for collaborative interventions should be explored.

Currently, tools to facilitate activation of the guidelines within schools have not been developed. Existing resources for teachers (i.e., PlaySport) must be adapted to harmonize with the new guidelines. Innovative tools that facilitate and cue activation of the guidelines in the school environment are needed (e.g, userfriendly digital content, motivational apps and social media trig- 
gers for teacher). It is essential that customized materials be developed for local school administrators (e.g., principles) to assist them with incorporating the integrated guidelines into their school's overall programming. Resources to support advocacy efforts calling for province and/or board-wide change in policy related to the school environment and curriculum are required.

\section{Health professionals}

\section{Dissemination}

Health professionals are considered an expert and credible source of physical activity information (Faulkner et al. 2016). As such, health care interactions provide an opportunity to lay the framework for lifelong healthy behavior among children and youth. It is critical that dissemination activities occur in this context. Passive dissemination strategies could be as simple as having posters, tear sheets, and digital content in pediatricians and family physicians' offices, medical clinics, and waiting rooms. Active dissemination would involve a member of the healthcare team speaking to their young patients and/or their parents to introduce them to the integrated guidelines. However, given that physical activity messages, let alone sedentary behaviour and sleep guidelines, are just beginning to penetrate the primary care landscape (Carson et al. 2013), explicit awareness-raising activities targeting health professionals are needed.

CSEP as the host organization for the EIMC program is wellpositioned to raise awareness among health professionals of the integrated guidelines. The EIMC National Advisory Council, which includes representatives from most health professional organizations, is a suitable channel for disseminating guideline materials to physicians, nurses, and physiotherapists. Moreover, seeking the endorsement of the integrated guidelines by these organizations and support for dissemination of the guidelines among their networks would increase the likelihood of guideline uptake among healthcare professionals (Graham and Harrison 2005). The "whole day matters" concept and the integrated guidelines also should be introduced through educational activities including formational training activities (e.g., undergraduate medical education), continuing education forums, and informational outreach using formats accessible to health care providers to facilitate uptake (e.g., digital/apps). EIMC has hosted Exercise in Primary Care workshops, which should be updated to include the integrated guidelines to cue practitioners to disseminate the guidelines through their practice. These workshops are accredited for continuing medical education credits, which improves uptake. Of importance, the strong evidence base and the rigorous guideline development process underpinning the integrated guidelines must be emphasized in all of these educational activities to assure guideline credibility (Tremblay et al. 2016b).

\section{Implementation}

Implementation of the guidelines within clinical practice entails the assessment of patients' movement behaviour and/or counselling patients about meeting the guidelines. For health professionals who currently do not implement any movement guidelines in their practice, the introduction of new guidelines provides impetus to adopt new clinical practice. For health professionals who currently utilize movement guidelines, they likely only focus on specific behaviours (e.g., physical activity, sleep, sedentary time) in isolation rather than the full 24-h spectrum. As such, it is important for practitioners to understand the new guidelines and update their practice accordingly. Assessments of movement behaviour should now query sleep habits and practices, in addition to physical activity and sedentary behaviour (e.g., the Physical Activity and Sedentary Behaviour Questionnaire (CSEP 2013)). Accordingly, EIMC's key message encouraging practitioners to assess physical activity in every visit, using a physical activity or "exercise vital sign" will need to evolve to reflect the new guidelines.
Currently, no new resources have been developed to support the implementation of the integrated guidelines in clinical practice. For the guidelines to be implemented effectively in practice, practitioners must be equipped with the necessary skills to assess and counsel patients. Formative training programs and continuing education courses should be explored as channels for training practitioners to develop these skills. Further, as health professional curricula are reviewed, the new guidelines should be incorporated into training, particularly pediatrics and family medicine. For implementation to be feasible and effective, policy changes are necessary to create a fee structure that provides incentive to implement and counsel on the guidelines. The release of the new integrated guidelines creates opportunity for new collaborations among professional groups with varied areas of expertise. It may benefit health professionals to expand their referral networks to include sleep, play, ergonomic, and activity experts.

\section{Physical activity, child/youth service, and community organizations}

\section{Dissemination}

Any organizations that work directly with young people should be made aware of the new guidelines. This includes after-school care programs; arts and recreation programs; youth organizations such as the YMCA/YWCA, Scouts Canada/Girl Guides of Canada, and Boys and Girls Clubs of Canada; as well as both national and provincial sport organizations, including coaches and community centres. These groups have an important role to play in disseminating the new guidelines given they are typically guided by a mandate of promoting the overall well-being of young people. These groups also have opportunities to share information with parents and caregivers. With the ability to reach children/youth and parents, these organizations are well suited to engage, at minimum, in passive dissemination strategies including distribution of print resources. However, these groups should also capitalize on opportunities for more active dissemination of the new guidelines. For example, youth sport organizations at every level should emphasize the importance of adequate sleep for sport performance, with both athletes and parents. After-school programs and youth clubs can reiterate the importance of reducing sedentary leisure time activities and promoting light physical activity, and this should be reflected in the activity options made available to participants.

The tear sheets, infographics, and online content that CSEP and ParticipACTION have developed are the primary resource available to support these organizations' dissemination activities. Programming staff and administrators would benefit from attending a CSEP-hosted Webinar regarding the guidelines.

\section{Implementation}

Child and youth service organizations are well-positioned to implement at least some aspects of the guidelines. Sport organizations, camps, and other clubs often focus on sports or active games, however, in reality much time is often spent having children sit to listen to instructions or waiting for their "turn" (e.g., Sacheck et al 2011). This time can be reduced by minimizing passive instruction time and not requiring (or allowing) participants to sit for these periods. Light activity such as walking or stretching can be incorporated into practices and games, in between bouts of more vigorous activity. On a policy level, sport groups should ensure that games and practice times are not held later in the evenings or too early in the mornings, to avoid encroaching on sleep time and consistency.

Given that young people spend a great deal of their day seated at school, youth clubs, camps, arts and recreation programs, and after-school programs should endeavour to get kids up and moving as much as possible during waking hours. This can be achieved by offering diverse activities to appeal to a range of young people, by incorporating time for active free play into pro- 
gramming, and by discouraging (or banning) the use of screens in these settings. While time and space availability may constrain opportunities for moderate to vigorous physical activity, replacing sedentary time with light activity is achievable in most spaces. Leaders within these groups should set a good example by minimizing screen time themselves and encouraging active participation in a range of activities.

Currently, no specific resources are developed to support the implementation of the integrated guidelines in physical activity, child/youth service, and community organizations. It should be a priority to develop materials for this important delivery segment. To ensure organizations can achieve their mandate and activate the integrated guidelines, these materials should be developed collaboratively by the lead disseminators and representatives from user groups.

\section{Movement, health, child development, and parent-centred organizations}

\section{Dissemination}

Intermediary groups including nongovernmental and governmental organizations with mandates to promote movement behaviours, health, and/or child development must be involved in disseminating the guidelines. Parent-centred organizations must also be engaged in dissemination. These intermediary groups include organizations such as the Canadian Sleep Society, the Sedentary Behaviour Research Network, the Public Health Agency of Canada, local public health units, the Canadian Public Health Association, PHE Canada, Active Canada 20/20, Heart and Stroke Foundation of Canada, the Canadian Diabetes Association, and the Chronic Disease Prevention Alliance of Canada. Workplaces also are an important parent- and guardian-centred intermediary group; for example, federal and provincial public service institutions, municipalities, and Conference Board of Canada members (many are large corporations) are options for targeted dissemination. Of note, the new guidelines present opportunity for the movement organizations in particular to review and refresh their strategic objectives, considering how their mandate aligns with the whole day approach.

With regard to these organizations' dissemination activities, the new guidelines must be incorporated into all relevant training, materials, and awareness activities. For some organizations dissemination could be as simple as updating materials provided to clients from the old physical activity guidelines to the new integrated movement guidelines. However, in many cases the release of the new guidelines will have a larger impact and may require updating an array of educational materials, including manuals and texts, changing assessment and prescription tools, and creating new professional development materials and opportunities. In doing so, organizations need to think creatively about how to engage their target audience in awareness and knowledge of the new guidelines. This requires novel approaches for interaction with the new material such as through social media, digital apps, blogs, Webinars, and in-person workshops could help engage their audience. Interactions with the population through the myriad of available physical activity monitoring technologies provide the potential to partner with technology manufacturers and/or insurance companies who collect and analyze these data. Moreover, they provide organizations the means to not only create novel learning opportunities, but also provide potential sources of evaluation of the effectiveness of uptake.

This transition to the new guidelines and development of novel approaches to engagement undoubtedly will take time and resources. At the very minimum, it is important for organizations to update their Website content; the Internet is a major source for health information (Beaudoin and Hong 2011). In the past, some organizations fully integrated new guidelines into their online resources, while others did not adopt the new resources and left old guidelines on their Websites (Gainforth et al. 2013). Organizations must make an effort to change the online content to ensure the public is presented with a current, unified message about movement behaviour recommendations. To further address the resource implications of changing the guidelines, organizations should seek out collaborations with organizations with expertise, reach, and/or resources that will complement theirs. These interdisciplinary and inter-sector collaborations will facilitate message cross-promotion between organizations, minimize duplication of efforts, and will result in a synergistic and holistic approach towards improving individual/family well-being.

CSEP and ParticipACTION are supporting these organizations' dissemination activities by updating their own Website content. In the absence of resources to change online content, organizations can direct users to this up-to-date information. They also can adopt any of the public-facing resources CSEP and ParticipACTION create.

\section{Implementation}

For many of these organizations, guideline implementation in the traditional sense of offering programs and services to children and youth that align with the guidelines is outside their scope. Nonetheless, these organizations have an important role in implementation. Several organizations will be integral in supporting the adoption of guidelines by other intermediary groups (e.g., PHE Canada for support for uptake and activation in schools; public health units for support for uptake and activation in clinical settings). Many of these organizations influence policy either by developing policy itself or in advocating for policy change. Current policies around physical activity and child health should be reviewed and updated to reflect the current state of science the integrated guidelines. Some of these organizations have a significant role in the surveillance of health behaviour among children and youth (e.g., Canadian Health Measures Survey, Canadian Community Health Survey, Canadian Physical Activity Levels Among Youth survey (CANPLAY), Health Behaviour of School-aged Children Survey). The new guidelines must be used to inform the measurement and analysis approaches used in these surveillance activities. New measurement techniques and additional survey items may need to be used to ensure guideline adherence can be determined. Other organizations such as the Heart and Stroke Foundation of Canada have influence on the research agenda through their funding activities. Through strategic funding priorities, these organizations can help to shift researchers' focus from 1 or 2 specific movement behaviours to the full movement spectrum. This shift will create avenues for new research questions, novel research designs, and cutting-edge analysis techniques. Several of these opportunities are highlighted throughout this issue. Examples of new analysis techniques and suggested surveillance procedures for the new integrated guidelines are provided elsewhere in the issue (Carson et al. 2016b; Tremblay et al. 2016b). The launch of new guidelines represents a natural experiment that should be evaluated. A process evaluation assessing how the guidelines are being disseminated and implemented and an outcome evaluation assessing the impact of dissemination and implementation activities on proximal (e.g., attitudes) and distal outcomes (e.g., behaviour) has been proposed (Tremblay et al. 2016a). The extent of evaluation that will be conducted depends on funding availability.

All of the articles in this supplement are a key resource available to support these organizations' implementation activities. They provide a strong, comprehensive evidence-base upon which to found policy change, strategic research planning and surveillance activities.

\section{Media}

\section{Dissemination}

Previous guideline launches have drawn significant media attention with over 100 million impressions. Media, once again, will 
play an integral role in launching, explaining, and promoting the new integrated guidelines. Educating members of the media during both the pre- and post-launch periods about this new integrated approach to thinking about movement behaviours will be critical to effective ongoing guideline dissemination. One of the challenges around communication will be to explain that these new integrated 24-h movement guidelines are for children and youth, and that a lag time (perhaps a significant one) is expected before similar guidelines are available for other population groups.

ParticipACTION is leveraging existing relationships with media outlets to communicate the new guidelines. The press package emphasizes the novelty of the integrated approach and provides recommendations for applying this approach to their ongoing coverage of stories related to physical activity, sedentary behaviour, and/or sleep for children and youth. Beyond this initial press package, all intermediary groups who interact with media are encouraged to reinforce the whole day matters concept.

\section{Transforming opportunity into reality}

The new 24-Hour Movement Guidelines for Children and Youth represent a step forward in how we promote healthy active living. The integration of sleep, sedentary behaviour, and all levels of physical activity highlights the positive interactive and potentially synergistic effects that can occur when behaviours are viewed in a more holistic way. This new paradigm presents a real opportunity to use the new guidelines in innovative ways and to reinvigorate the conversation about active living. Capitalizing on this momentum for change, practitioners, professionals, and organizations are called to not only disseminate these new guidelines but to implement them in their practice. For this potential to be fully realized, however, adequate funding is essential to support creative, novel, and collaborative strategies for maximizing uptake and ensuring activation. In the absence of this, guideline launch activities will not extend much beyond the short-term dissemination activities currently planned - despite the apparent need for implementation efforts. It is only through well-funded, collective engagement in disseminating and implementing these new guidelines that substantive changes can be made in the actual behaviours of the population. The time is now to push forward in how we address health behaviours. Given the dismal outlook of declining fitness and health of our children and youth (Tremblay et al. 2010) and poor prognosis for healthcare budgets, our future depends on it.

\section{Conflict of interest statement}

The authors declare that there are no conflicts of interest.

\section{References}

Beaudoin, C.E., and Hong, T. 2011. Health information seeking, diet and physical activity: an empirical assessment by medium and critical demographics. Int. J. Med. Inform. 80(8): 586-595. doi:10.1016/j.ijmedinf.2011.04.003. PMID: 21640643

Brawley, L.R., and Latimer, A.E. 2007. Physical activity guides for Canadians: messaging strategies, realistic expectations for change, and evaluation. Appl. Physiol. Nutr. Metab. 32(S2E): S170-S184. doi:10.1139/H07-105.

Canadian Society for Exercise Physiology. 2013. Canadian Society for Exercise Physiology-Physical Activity Training for Health (CSEP-PATH). CSEP, Ottawa, Ont., Canada.

Carson, V., LeBlanc, C., Moreau, E., and Tremblay, M.S. 2013. Paediatricians' awareness of, agreement with, and use of the new Canadian Physical Activity and Sedentary Behaviour Guidelines for ages 0-17 years. Paediatr. Child Health, 18(10): 538-542. PMID:24497781.

Carson, V., Hunter, S., Kuzik, N., Gray, C.E., Poitras, V.J., Chaput, J.-P., et al. 2016a. Systematic review of sedentary behaviour and health indicators in schoolaged children and youth: an update. Appl. Physiol. Nutr. Metab. 41: This issue. doi:10.1139/apnm-2015-0630.

Carson, V., Tremblay, M.S., Chaput, J.-P., and Chastin, S.F.M. 2016b. Associations between sleep duration, sedentary time, physical activity and health indicators among Canadian children and youth using compositional analyses. Appl. Physiol. Nutr. Metab. 41: This issue. doi:10.1139/apnm-2016-0026.

Chaput, J.-P., Carson, V., Gray, C., and Tremblay, M.S. 2014. Importance of all movement behaviors in a 24 hour period for overall health. Int. J. Environ. Res. Public Health, 11: 12575-12581. doi:10.3390/ijerph111212575. PMID:25485978.

Chaput, J.-P., Gray, C.E., Poitras, V.J., Carson, V., Gruber, R., Olds, T., et al. 2016. Systematic review of the relationships between sleep duration and health indicators in school-aged children and youth. Appl. Physiol. Nutr. Metab. 41: This issue. doi:10.1139/apnm-2015-0627.

Colley, R.C., Garriguet, D., Janssen, I., Craig, C.L., Clarke, J., and Tremblay, M.S. 2011. Physical activity of Canadian children and youth: accelerometer results from the 2007 to 2009 Canadian Health Measures Survey. Health Rep. 22(1): 15-24. PMID:21510586.

Colley, R.C., Wong, S.L., Garriguet, D., Janssen, I., Connor Gorber, S., and Tremblay, M.S. 2012. Physical activity, sedentary behaviour and sleep in Canadian children: parent-report versus direct measures and relative associations with health risk. Health Rep. 23(2): 45-52. PMID:22866540.

Faulkner, G., White, L., Riazi, N., Latimer-Cheung, A.E., and Tremblay, M.S. 2016. Canadian 24-Hour Movement Guidelines for Children and Youth: Exploring the perceptions of stakeholders regarding their acceptability, barriers to uptake, and dissemination. Appl. Physiol. Nutr. Metab. 41: This issue. doi:10. 1139/apnm-2016-0100.

Gainforth, H.L., Berry, T., Faulkner, G., Rhodes, R.E., Spence, J.C., Tremblay, M.S., and Latimer-Cheung, A.E. 2013. Evaluating the uptake of Canada's new physical activity and sedentary behavior guidelines on service organizations' websites. Transl. Behav. Med. 3(2): 172-179. doi:10.1007/s13142-012-0190-z. PMID: 24073168.

Graham, I.D., and Harrison, M.B. 2005. Evaluation and adaptation of clinical practice guidelines. Evid.-Based Nurs. 8(3): 68-72. doi:10.1136/ebn.8.3.68. PMID:16021701.

Harris, J.R., Cheadle, A., Hannon, P.A., Forehand, M., Lichiello, P., Mahoney, E., et al. 2012. A framework for disseminating evidence-based health promotion practices. Prev. Chronic Dis. 9: 110081. doi:10.5888/pcd9.110081.

Larouche, R., Saunders, T.J., Faulkner, G.E.J., Colley, R., and Tremblay, M.S. 2014. Associations between active school transport and physical activity, body composition, and cardiovascular fitness: a systematic review of 68 studies. J. Phys. Act. Health, 11(1): 206-227. PMID:23250273.

LeBlanc, A.G., Berry, T., Deshpande, S., Duggan, M., Faulkner, G., Latimer-Cheung, A.E., et al. 2015. Knowledge and awareness of Canadian Physical Activity and Sedentary Behaviour Guidelines: a synthesis of existing evidence. Appl. Physiol. Nutr. Metab. 40(7): 716-724. doi:10.1139/apnm-2014-0464. PMID:26099846.

Louis, M.R., and Sutton, R.I. 1991. Switching cognitive gears: from habits of mind to active thinking. Hum. Relat. 44: 55-76. doi:10.1177/001872679104400104.

McGuire, W.J. 1984. Public communication as a strategy for inducing healthpromoting behavioral change. Prev. Med. 13: 299-319. doi:10.1016/00917435(84)90086-0. PMID:6387698.

Michie, S., van Stralen, M.M., and West, R. 2011. The behaviour change wheel: a new method for characterising and designing behaviour change interventions. Implement. Sci. 6: 42. doi:10.1186/1748-5908-6-42. PMID:21513547.

ParticipACTION. 2016. 2016 ParticipACTION Report Card on the Physical Activity of Children and Youth. ParticipACTION, Toronto, Ont., Canada.

Poitras, V.J., Gray, C.E., Borghese, M.M., Carson, V., Chaput, J.-P., Janssen, I., et al. 2016. Systematic review of the relationships between objectively measured physical activity and health indicators in school-aged children and youth. Appl. Physiol. Nutr. Metab. 41: This issue. doi:10.1139/apnm-2015-0663.

Sacheck, J.M., Nelson, T., Ficker, L., Kafka, T., Kuder, J., and Economos, C.D. 2011. Physical activity during soccer and its contribution to physical activity recommendations in normal weight and overweight children. Pediatr. Exerc. Sci. 23(2): 281-292. PMID:21633140.

Saunders, T.J., Gray, C.E., Poitras, V.J., Chaput, J.-P., Janssen, I., Katzmarzyk, P.T., et al. 2016. Combinations of physical activity, sedentary behaviour and sleep: relationships with health indicators in school-aged children and youth. Appl. Physiol. Nutr. Metab. 41: This issue. doi:10.1139/apnm-2015-0626.

Stone, M.R., Faulkner, G.E., Zeglen-Hunt, L., and Bonne, J.C. 2012. The Daily Physical Activity (DPA) policy in Ontario: is it working? An examination using accelerometry-measured physical activity data. Can. J. Publ. Health, 103(3): 170-174. PMID:22905633.

Tremblay, M.S., Shields, M., Laviolette, M., Craig, C.L., Janssen, I., and Connor Gorber, S. 2010. Fitness of Canadian children and youth: Results from the 2007-2009 Canadian Health Measures Survey. Health Rep. 21(1): 7-20. PMID:20426223.

Tremblay, M.S., Barnes, J.D., and Cowie Bonne, J. 2014. Impact of the Active Healthy Kids Canada Report Card: a 10-year analysis. J. Phys. Act. Health, 11(S1): S3-S20. PMID:25426911.

Tremblay, M.S., Carson, V., Chaput, J.-P., Gorber, S.C., Dinh, T., Duggan, M., et al. 2016a. Canadian 24-Hour Movement Guidelines for Children and Youth: An integration of physical activity, sedentary behaviour, and sleep. Appl. Physiol. Nutr. Metab. 41: This issue. doi:10.1139/apnm-2016-0151.

Tremblay, M.S., Carson, V., and Chaput, J.-P. 2016b. Introduction to the Canadian 24-Hour Movement Guidelines for Children and Youth: An integration of physical activity, sedentary behaviour, and sleep. Appl. Physiol. Nutr. Metab. 41: This issue. doi:10.1139/apnm-2016-0203. 